\title{
Brief Review on Nano Robots in Bio Medical Applications M Sivasankar ${ }^{1 *}$ and RB Durairaj ${ }^{2}$
}

${ }^{1}$ Arunai Engineering College, Velu Nagar, Mathur, Tiruvannamalai, Tamilnadu, India

2SRM University, Chennai, Tamilnadu, India

\begin{abstract}
Nano-robots are the robots that are simply known as that controllable machine at the nano $\left(10^{-9}\right)$ meter or molecular scale, composed of nano-components. More specifically, nano robotics refers to the still largely hypothetical nanotechnology engineering discipline of designing and building nano robots. Even though the field of nano robotics is fundamentally different from that of the macro robots due to the differences in scale and material, there are many similarities in design and control techniques that eventually could be projected and applied. Due to the modern scientific capabilities, it has become possible to attempt the creation of nano robotic devices and interface them with the macro world for control. There are countless such machines which exist in nature and there is an opportunity to build more of them by mimicking nature. Nowadays these nano robots play a vital role in the field of Bio Medicine. Especially in the treatment of cancer, Cerebral Aneurysm, kidney stones removal, also to remove the defected part in our DNA structure and some other treatments that has the greatest aid to save human lives. This paper guides to the recent research on nano robots in the Bio medical applications.
\end{abstract}

Keywords: Nano robots; Cerebral aneurysm; Nanotechnology: Biomedicine; Nano-components

\section{Introduction}

Nanotechnology can best be defined as a description of activities at the level of atoms and molecules that have applications in the real world. A nanometer is a billionth of a meter, that is, about $1 / 80,000$ of the diameter of a human hair, or 10 times the diameter of a hydrogen atom. The size-related challenge is the ability to measure, manipulate and assemble matter with features on the scale of 1-100 nm. In order to achieve cost-effectiveness in nanotechnology it will be necessary to automate molecular manufacturing. The engineering of molecular products needs to be carried out by robotic devices, which have been termed nano robots [1]. This review chapter focuses on the state of the art in the emerging field of nano robotics, its applications and discusses in brief some of the essential properties and dynamical laws which makes this field more challenging and unique than its macro scale counterpart. Some proponents of nano robotics, in reaction to the grey goo scare scenarios, hold the view that nano robots capable of replication outside of a restricted factory environment do not form a necessary part of a purported productive nanotechnology and that the process of self-replication, if it were ever to be developed, could be made inherently safe. They further assert that their current plans for developing and using molecular manufacturing do not in fact include free-foraging replicator. Nano robots with complete artificial components have not been realized yet. The active area of research in this field is focused more on molecular robots, which are thoroughly inspired by nature's way of doing things at nano scale [2]. Mother Nature has her own set of molecular machines that have been working for centuries and have been optimized for performance and design over the ages. As our knowledge and understanding of these numerous machines continues to increase, we now see a possibility of using the natural machines, or creating synthetic ones from scratch, using nature's components. The main goal in the field of molecular machines is to use various biological elements whose function at the cellular level creates motion, force or a signal as machine components. These components perform their preprogrammed biological function in response to the specific physiochemical stimuli but in an artificial setting. In this way proteins and DNA could act as motors, mechanical joints, transmission elements, or sensors. If all these different components were assembled

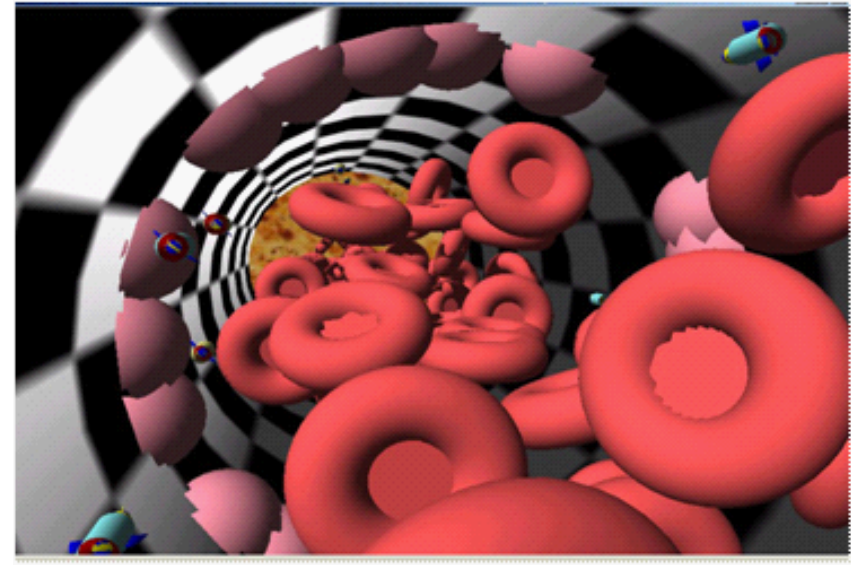

Figure 1: Nano Robots Designed for reduction of atherosclerotic lesion in heart.

together in the proper proportion and orientation they would form nano devices with multiple degrees of freedom, able to apply forces and manipulate objects in the nano scale world. The advantage of using nature's machine components is that they are highly efficient and reliable (Figure 1).

\section{Design of nano robots}

The software NCD (nano robot control design) is a system

*Corresponding author: Dr. M Sivasankar, Professor and Director of Bio-Medica research, Arunai Engineering College, Velu Nagar, Mathur, Tiruvannamalai, Tamilnadu, India, E-mail: dr.biosankar@gmail.com

Received December 13, 2012; Accepted February 25, 2012; Published February 27, 2012

Citation: Sivasankar M, Durairaj RB (2012) Brief Review on Nano Robots in Bio Medical Applications.]- L\$ GY5 RERM\$ XIRP DW1:101. doi:10.4172/2168-9695.1000101

Copyright: ( 2012 Sivasankar M. This is an open-access article distributed under the terms of the Creative Commons Attribution License, which permits unrestricted use, distribution, and reproduction in any medium, provided the original author and source are credited. 
implemented to serve as a test bed for nano robot $3 \mathrm{D}$ prototyping, serving as a fast development platform for medical nano robots investigation, the NCD simulations show how to interact and control a nano robot inside the body. It is an advanced nano mechatronics simulator that provides physical and numerical information for nano robot task-based modeling. The nano robot design is comprised of integrated nano electronics and components such as molecular sorting rotors and a robot arm (telescoping manipulator) derived from biological models. The nano robot exterior shape consists of a diamondoid material to which may be attached an artificial glycocalyx surface that minimizes fibrinogen (and other blood proteins) adsorption and bioactivity, ensuring sufficient biocompatibility to avoid immune system attack. Different molecules types are distinguished by a series of chemotactic sensors whose binding sites have a different affinity for each kind of molecule. These sensors also detect obstacles which might require a new trajectory planning. Sensor design and capabilities depend on the details of the environment and task. Thus, the nano robot requires transducers capabilities and smart sensors directly related to that specific biomedical application. In present study, the nano robot is able to detect obstacles over a range of about $1 \mathrm{~mm}$ and within an angular resolution equivalent to a diameter of $100 \mathrm{~nm}$ at that range. The biomolecules are too small to be detected reliably: instead the robot relies on chemical contact sensors to detect them. This description of interaction capabilities allows evaluating different nano robot sensor based action [3]. It also helps to choose the kind of low-level control to maximize the information acquired for an effective real time performance. The nano robot kinematics can be predicted using state equations, positional constraints, inverse kinematics and dynamics, while some individual directional component performance can be simulated using control system models of transient and steadystate response.

\section{Medical application of nano robots}

Nano robots are expected to enable new treatments for patients suffering from different diseases and will result in a remarkable advance in the history of medicine. Recent developments in the field of bimolecular computing, this is a promising first step to enable future nano processors with increased complexity. Studies targeted at building biosensors and nano-kinetic devices required to enable medical nano robotics operation and locomotion, have also been progressing [4]. The use of nano robots may advance biomedical intervention with minimally invasive surgeries and help patients who need constant body functions monitoring, or ever improve treatments efficiency through early diagnosis of possible serious diseases [5]. For example, the nano robots may be utilized to attach on transmigrating inflammatory cells or white blood cells, thus reaching inflamed tissues faster to assist in their healing process. Nano robots will be applied in chemotherapy to combat cancer through precise chemical dosage administration and a similar approach could be taken to enable nano robots to deliver anti-HIV drugs. Nano robots could be used to process specific chemical reactions in the human body as ancillary devices for injured organs. Monitoring diabetes and controlling glucose levels for patients will be a possible application of nano robots [6]. Nano robots might be used to seek and break kidney stones. Another important possible feature of medical nano robots will be the capability to locate atherosclerotic lesions in stenosed blood vessels, particularly in the coronary circulation and treat them either mechanically, chemically or pharmacologically. Organic nano robots are the work on ATP and DNA based molecular machines, also known as bio-nanorobots [7]. In this case the idea is the development of ribonucleic acid and adenosine triphosphate devices and even the use of modified microorganisms to achieve some kind of bio molecular computation, sensing and actuation for nano robots. Another approach for the development of molecular machines is the inorganic nano robot. Inorganic nano robots development is based on tailored nano electronics. In comparison with bionanorobots, inorganic nanorobots could achieve a considerably higher complexity of integrated nano scale components. There are some works on how to enable manufacturing of inorganic nano robots. The use of new diamondoid rigid materials is a possible approach that may help towards achieving new materials for inorganic nano robots. The approach taken in our work is the NanoBuild Hardware Integrated System (Nanobhis) [8,9]. It represents a joint set of well established techniques and new methodologies from nanotechnology with the aim of manufacturing nano robots [10]. It is used 3D simulation and manufacturing design with integrated nano electronics analyses. The challenge of manufacturing nano robots may result from new methodologies in fabrication, computation, sensing and manipulation Real time 3D prototyping tools are import to help on nanotechnology development. It may have a direct impact on the implementation of the new approaches for manufacturing techniques. Simulation can anticipate performance of new nano devices. Further, it can also help on nano mechatronics design and in testing control and automation strategies.

\section{Nano robots in cancer treatment}

Cancer can be successfully treated with current stages of medical technologies and therapy tools. However, a decisive factor to determine the chances for a patient with cancer to survive is: how earlier it was diagnosed; what means, if possible, a cancer should be detected at least before the metastasis has began [11]. Another important aspect to achieve a successful treatment for patients is the development of efficient targeted drug delivery to decrease the side effects from chemotherapy. Considering the properties of nano robots to navigate as blood borne devices, they can help on such extremely important aspects of cancer therapy. Nanorobots with embedded chemical biosensors can be used to perform detection of tumour cells in early stages of development inside the patient's body [12]. Integrated nano sensors can be utilized for such a task in order to find intensity of E-cadherin signals. Therefore a hardware architecture based on nano bioelectronics is described for the application of nanorobots for cancer therapy. Analyses and conclusions for the proposed model are obtained through real time 3D simulation.

\section{Nano robots in gene therapy}

Medical nano robots can readily treat genetic diseases by comparing the molecular structures of both DNA and proteins found in the cell to known or desired reference structures. Any irregularities can then be corrected, or desired modifications can be edited in place. In some cases, chromosomal replacement therapy is more efficient than in $\mathrm{CY}$ to repair. Floating inside the nucleus of a human cell, an assemblerbuilt repair vessel performs some genetic maintenance. Stretching a super coil of DNA between its lower pair of robot arms, the nano machine gently pulls the unwound strand through an opening in its prow for analysis. Upper arms, meanwhile, detach regulatory proteins from the chain and place them in an intake port $[13,14]$. The molecular structures of both DNA and proteins are compared to information stored in the database of a larger nano computer positioned outside the nucleus and connected to the cell-repair ship by a communications link. Irregularities found in either structure are corrected and the proteins reattached to the DNA chain, which re-coils into its original form with a diameter of only 50 nanometers, the repair vessel would be smaller than most bacteria and viruses, yet capable of therapies and cures well beyond the reach of present-day physicians. With trillions 
of these machines coursing through a patient's bloodstream, "internal medicine" would take on new significance. Disease would be attacked at the molecular level and such maladies as cancer, viral infections and arteriosclerosis could be wiped out.

\section{Nano robot for brain aneurysm}

The nano robot for brain aneurysm prognosis, using computational nanotechnology for medical device prototyping. This is consist of three main aspects are Equipment prototyping, the manufacturing approach and inside-body transduction. Equipment prototyping is the computational nanotechnology provides a key tool for the fast and effective development of nano robots, helping in the investigation to address major aspects on medical instrumentation and device prototyping. A similar approach was previously taken by industry to build racing cars, airplanes, submarines, ICs and medical devices [15]. Now, the same can be used to benefit the development and research of medical nano robots [16-18]. The Manufacturing technology for manufacturing purposes of the nano robot should be integrated as a biochip device. Thus, new materials, photonics and nano bioelectronics are presented with a description of the nano robot architecture. And the Inside-body transductions are cell morphology, microbiology and proteomics are used as parameters for nano robot morphology and inside-body interaction. Changes on chemical gradients and telemetric instrumentation are used for medical prognosis, with the nano robots activation based on proteomic over expression [19]. As presented in the paper, these three points comprise the key pieces required to advance the development and implementation of medical nano robotics. For brain aneurysm prognosis, nano robots need to track the vessel endothelial injury before a subarachnoid hemorrhage occurs. These changes on chemical concentration are used to guide the nano robots to identify brain aneurysm in the early stages of development (Figure 2).

The bio molecules are too small to be detected reliably: instead the robot relies on chemical nano biosensor contact to detect them [20]. The main morphologic aspects related to brain aneurysm are taken for modeling the study of nano robots sensing and interaction within the deformed blood vessel $[21,22]$. Intracranial concentrations of NOS are small and some false positives can even occur due to some positive functions of N-oxide with semi carbazone (pNOS). Cells and nano robots continually enter one end of the workspace along with the fluid flow. The nano robots must detect protein over expression and the setup for sensing and control activation can be modified for different values, such as adjusting the detection thresholds. We treat any nano robots not responding while within the workspace as if they did not detect any signal, so they flow with the fluid as it leaves the workspace. If the nano robot's electrochemical sensor detects NOS in low quantities or inside normal gradient it generates a weak signal lower than $50 \mathrm{nA}$ [23-25]. In such a case the nano robot ignores the NOS concentration assuming that it is within the expected levels of intracranial NOS. As a practical threshold for medical prognosis, to avoid noise distortions and achieve a higher resolution, each time the cell phone has received at least a total of 100 nano robots higher proteomic signal transduction, the model considers this as strong evidence of intracranial aneurysm. When activated, the nano robots' sensors also indicate their respective position at the moment that they detected a high NOS protein concentration providing useful information about the vessel bulb location and dimensions.

\section{Nano robots in dentistry}

The growing interest in the future of dental applications of nanotechnology is leading to the emergence of a new field called Nano dentistry. Nano robots induce oral analgesia, Desensitize tooth; manipulate the tissue to re-align and straighten irregular set of teeth and to improve durability of teeth. Further it is explained that how nano robots are used to do preventive, restorative, curative procedures [26,27]. Nano dental techniques involve many tissue engineering procedures for major tooth repair. Mainly nano robotics manufacture and installation of a biologically autologous whole replacement tooth that includes both mineral and cellular components which leads to complete dentition replacement therapy. Nano dentistry has given material that is nano structured composite material, sapphire which increases tooth durability and appearance. Upper enamel layers are replaced by covalently bonded artificial material such as sapphire [28] This material has 100 to 200 times the hardness and failure strength than ceramic. Like enamel, sapphire is a somewhat susceptible to acid corrosion [29]. Sapphire has best standard whitening sealant, cosmetic alternative. New restorative nano material to increase tooth durability is Nano composites. This is manufactured by nano agglomerated discrete nano particles that are homogeneously distributed in resins or coatings to produce nano composites [30,31]. The nano filler include an alumino silicate powder having a mean particle size of about $80 \mathrm{~nm}$ and a $1: 4$ ratio of alumina to silica. The nano filler has a refractive index of 1.503; it has superior hardness, modulus of elasticity, translucency, esthetic appeal, excellent color density, high polish and 50\% reduction in filling shrinkage. They are superior to conventional composites and blend with a natural tooth structure much better.

\section{Conclusion}

Nanotechnology as a diagnostic and treatment tool for patients
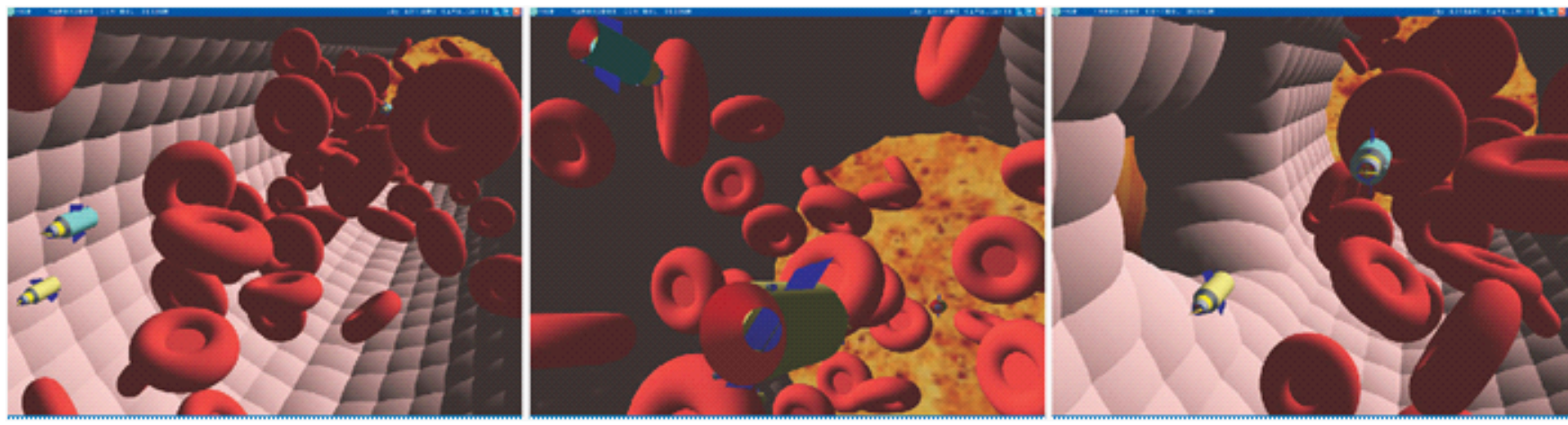

Figure 2: Working of Nanorobot on Brain aneurysm. 
with cancer and diabetes showed how actual developments in new manufacturing technologies are enabling innovative works which may help in constructing and employing nano robots most effectively for biomedical problems. Nano robots applied to medicine hold a wealth of promise from eradicating disease to reversing the aging process (wrinkles, loss of bone mass and age-related conditions are all treatable at the cellular level); nano robots are also candidates for industrial applications. They will provide personalized treatments with improved efficacy and reduced side effects that are not available today. They will provide combined action drugs marketed with diagnostics, imaging agents acting as drugs, surgery with instant diagnostic feedback. The advent of molecular nanotechnology will again expand enormously the effectiveness, comfort and speed of future medical treatments while at the same time significantly reducing their risk, cost and invasiveness. This science might sound like a fiction now, but Nano robotics has strong potential to revolutionize healthcare, to treat disease in future. It opens up new ways for vast, abundant research work. Nanotechnology will change health care and human life more profoundly than other developments. Consequently they will change the shape of the industry, broadening the product development and marketing interactions between Pharma, Biotech, Diagnostic and Healthcare industries. Future healthcare will make use of sensitive new diagnostics for an improved personal risk assessment. Highest impact can be expected if those major diseases are addressed first, which impose the highest burden on the aging population: cardiovascular diseases, cancer, musculoskeletal conditions, neurodegenerative and psychiatric diseases, diabetes and viral infections. Nano medicine holds the promise to lead to an earlier diagnosis, better therapy and improved follow up care, making the health care more effective and affordable. Nano medicine will also allow a more personalized treatment for many diseases, exploiting the in-depth understanding of diseases on a molecular level.

\section{References}

1. Nanorobotics By ummat A, Dubey.A, Sharma.G, Mavroidis Department of Mechanical and Industrial Engineering, Northeastern University ,. Department of Mechanical and Aerospace Engineering, Rutgers University.

2. Wang J (2009) "Can Man-Made Nanomachines Compete with Nature Biomotors?". ACS Nano 3: 4-9.

3. Cale TS, LuJ-Q, Gutmann RJ (2008) "Three-dimensional integration in microelectronics: Motivation, processing, and thermomechanical modeling". Chemical Engineering Communications 195: 847-888.

4. Couvreur P, Vauthier C (2006) "Nanotechnology: Intelligent Design to Treat Complex Disease". Pharmaceutical Research 23: 1417-1450.

5. Cavalcanti A, Freitas Jr RA, Kretly LC (2004) "Nanorobotics Control Design: A Practical Approach Tutorial". In Proc ASME 28th Biennial Mechanisms and Robotics Conference, Salt Lake City, Utah, USA. ,

6. Cui Y, Wei Q, Park H, Lieber CM (2001) Nanowire nanosensors for highly sensitive and selective detection of biological and chemical species. Science 293: 1289-1292.

7. Robert A, Freitas Jr (2005) Current Status of Nanomedicine and Medical Nanorobotics. J Comput Theor Nanosci 2: 1-25.

8. Cavalcanti A, Shirinzadeh B, Freitas RA Jr, Hogg T (2008) Nanorobot architecture for medical target identification. Nanotechnology 19: 015103.

9. Cavalcanti A, Shirinzadeh B, Zhang M, Kretly LC (2008) Nanorobot hardware architecture for medical defense. Sensors 8: 2932-2958.

10. Chau R, Doyle B, Datta S, Kavalieros J, Zhang K (2007). Integrated nanoelectronics for the future. Nature Materials 6: 810-812.
11. Fisher B (2008) "Biological Research in the Evolution of Cancer Surgery: A Personal Perspective". Cancer Research 68: 10007-10020.

12. Hill C, Amodeo A, Joseph JV, Patel HRH (2008) "Nano- and microrobotics: how far is the reality?". Expert Review of Anticancer Therapy 8: 1891-1897.

13. Adleman LM (1996) "On Constructing A Molecular Computer", DNA Based Computers II: Dimacs Workshop, Jun. 10-12, (Dimacs Series in Discrete Mathematics and Theoretical Computer Science, V. 44), American Mathematical Society 1-21.

14. Hamdi M, Ferreira A, Sharma G, Mavroidis C (2008) Prototyping bio-nanorobots using molecular dynamics simulation and virtual reality. Microelectronics Journal 39:190-201.

15. Genov R, Stanacevic M, Naware M, Cauwenberghs G, Thakor NV (2006) 16-Channel integrated potentiostat for distributed neurochemical sensing. IEEE Transactions on Circuits and Systems I-Regular Papers 53: 2371-2376.

16. Cavalcanti (2003) Assembly Automation with Evolutionary Nanorobots and Sensor-Based Control Applied to Nanomedicine". IEEE Transactions on Nanotechnology 2: 82-87.

17. Hogg T (2007) Coordinating microscopic robots in viscous fluids. Auton Agent Multi Agent Syst 14: 271-305

18. Cavalcanti A, Freitas Jr RA (2002) "Autonomous Multi- Robot Sensor-Based Cooperation for Nanomedicine", Int'I J. Nonlinear Science Numerical Simulation 3: 743-746.

19. Buchanan JR, Kleinstreuer C (1998) "Simulation of Particle-Hemodynamics in a Partially Occluded Artery Segment with Implications to the Initiation of Microemboli and Secondary Stenoses". J Biomech Eng 120: 446-454.

20. Casal A, Hogg T, Cavalcanti A (2003) "Nanorobots as Cellular Assistants in Inflammatory Responses", in Proc. IEEE BCATS Biomedical Computation at Stanford 2003 Symposium, IEEE Computer Society, Stanford CA, USA.

21. Elder JB, Hoh DJ, Oh BC, Heller AC, Liu CY, et al. (2008) "The future of cerebra surgery: a kaleidoscope of opportunities”. Neurosurgery 62: 1555-1582.

22. Elder JB, Liu CY, Apuzzo MLJ (2008) Neurosurgery in the realm of 10_9, Part 2: Applications of nanotechnology to neurosurgery-present and future. Neurosurgery 62: 269-285.

23. Freitas RA Jr (2005) What is nanomedicine? Nanomedicine: Nanotechnology Biology and Medicine 1: 2-9.

24. Fukuda S, Hashimoto N, Naritomi H, Nagata I, Nozaki K, et al. (2000) Prevention of rat cerebral aneurysm formation by inhibition of nitric oxide synthase. Circulation 101: 2532-2538.

25. Fukuda T, Kawamoto A, Arai F, Matsuura H (1995). Steering mechanism and swimming experiment of micro mobile robot in water. Micro Electro Mechanical Systems 300-305

26. Cavalcanti A (2003) Assembly automation with evolutionary nanorobots and sensor-based control applied to nanomedicine. IEEE Transactions on Nanotechnology 2: 82-87.

27. Martel S, Mohammadi M, Felfoul O, Lu Z, Pouponneau P (2009). "Flagellated Magnetotactic Bacteria as Controlled MRItrackable Propulsion and Steering Systems for Medical Nanorobots Operating in the Human Microvasculature". International Journal of Robotics Research 28: 571-582.

28. Cavalcanti A (2009) "Nanorobot Invention and Linux: The Open Technology Factor - An Open Letter to UNO General Secretary”. CANNXS Project 1.

29. Vaughn JR (2006) "Over the Horizon: Potential Impact of Emerging Trends in Information and Communication Technology on Disability Policy and Practice". National Council on Disability 1-55.

30. Drexler KE (1992) "Nanosystems: molecular machinery, manufacturing, and computation". Wiley \& Sons.

31. Fishbine G (2001) “The investor's guide to nanotechnology \& micromachines". Wiley \& Sons.

32. FLUENT, Fluent Inc.

33. Freitas Jr RA (1999) "Nanomedicine, Vol. I: Basic Capabilities", Landes Bioscience. 